Pacific Journal of Mathematic 


\title{
OSCILLATORY PROPERTIES OF A DELAY DIFFER- ENTIAL EQUATION OF EVEN ORDER
}

\author{
RAYMOND D. TERRY
}

\begin{abstract}
A classification of nonoscillatory solutions according to the sign properties of their derivatives is introduced for a general nonlinear delay differential equation of order $2 n$. It is seen that there are $n$ types of positive solutions of this equation. An intermediate Riccatti transformation is employed to obtain integral criteria for the nonexistence of such solutions and for the oscillation of all solutions. Analysis of the Taylor Remainder gives rise to a summability condition which is used to investigate the asymptotic behavior of a class of solutions. The major results are then shown to be special cases of a more general result based on the direct method of Lyapunov.
\end{abstract}

The purpose of this paper is to discuss the oscillatory and nonoscillatory behavior of solutions of the nonlinear delay differential equation of order $2 n$ :

$$
D^{n}\left[r(t) D^{n} y(t)\right]+y_{\tau}(t) f\left(t, y_{\tau}(t)\right)=0,
$$

where $y_{\tau}(t)=y[t-\tau(t)], 0 \leqq \tau(t) \leqq T$ and $0<m \leqq r(t) \leqq M$. Throughout the first three sections $f(t, u)$ is assumed to satisfy the following three hypotheses:

(i) $f(t, u)$ is a continuous real-valued function on $[0, \infty) \times R$, $R=(-\infty, \infty)$

(ii) for each fixed $t \in[0, \infty), f(t, u)<f(t, v)$ for $0<u<v$; and

(iii) for each fixed $t \in[0, \infty), f(t, u)>0$ and $f(t, u)=f(t,-u)$ for $u \neq 0$.

In section four, these assumptions on $f(t, u)$ will be replaced by others as indicated there.

1. A solution $y(t)$ of $(1.1)$ is said to be oscillatory on $[0, \infty)$ if for each $t_{0}>0$, there exists a $T_{0}>t_{0}$ such that $y\left(T_{0}\right)=0$; it is called nonoscillatory otherwise. Following Kiguradze [4], we say that a solution $y(t)$ is of type $A_{j}$ if for sufficiently large $t$ the derivatives $D^{k} y(t)>0, k=0,1, \cdots, 2 j+1$ and $(-1)^{k+1} D^{k} y(t)>0, k=2 j+2, \cdots$, $2 n-1$. In an analogous manner we say that a solution $y(t)$ is of type $B_{j}$ if for sufficiently large $t, y_{k}(t)>0$ for $k=0, \cdots, 2 j+1$ and $(-1)^{k+1} y_{k}(t)>0, k=2 j+2, \cdots, 2 n-1$ where

and

$$
y_{k}(t)=D^{k} y(t), \quad k=0, \cdots, n-1
$$




$$
y_{k}(t)=D^{k-n}\left[r(t) D^{n} y(t)\right], \quad k=n, \cdots, 2 n-1 .
$$

When $r(t) \equiv 1$, these definitions reduce to those of types $A_{j}(0 \leqq j \leqq$ $n-1)$ respectively. In [4] Kiguradze proved a fundamental lemma which we state here as follows.

LEMMA 1.1. Let $u(t)$ be a continuous nonnegative function on $(0, \infty)$ having continuous derivatives up to order $2 n$ inclusive which do not change sign on this interval. If $D^{2 n} u(t) \leqq 0$, then there exists an integer $p=0, \cdots, n-1$ such that

$$
\begin{aligned}
D^{k} u(t) & \geqq 0, \quad k=0, \cdots, l, \\
(-1)^{k+1} D^{k} u(t) \geqq 0, & k=l+1, \cdots, 2 n-1,
\end{aligned}
$$

where $l=2 p+1 . \quad$ Furthermore, $0 \leqq D^{l} u(t) \leqq l ! t^{-l} u(t)$.

In view of this result, all nonoscillatory solutions of (1.1) with $r(t) \equiv 1$ are of type $A_{j}$ for some $j=0, \cdots, n-1$. In the general case we argue as follows. Suppose $y(t)$ is a nonoscillatory solution of (1.1) which we may assume to be nonnegative because of (iii). First, no two successive $y_{k}, k \geqq 1$ can ultimately be negative. Suppose $k \geqq n+1$ and $y_{k}$ and $y_{k+1}$ are negative for $t>a$. Then $y_{k}$ is a negative decreasing function on $[a, \infty)$ and there exists a constant $C_{k}>0$ such that $y_{k}(t)<-C_{k}$ for $t>a$. Thus

$$
y_{k-1}(t)-y_{k-1}(a)=\int_{a}^{t} y_{k}(s) d s<-C_{k}(t-a),
$$

which implies that $y_{k-1}$ is eventually negative. Next, if $y_{n}$ and $y_{n+1}$ are eventually negative, say for $t>a$, then

$$
\left[M y_{n-1}(s)\right]_{a}^{t}=M \int_{a}^{t} D^{n} y(s) d s<\int_{a}^{t} y_{n}(s) d s<-C_{n}(t-a),
$$

which implies that $y_{n-1}$ is eventually negative. Since $r(t)>0$ and $y_{n}(t)<0, D^{n} y(t)<0$. Using the negativity of $D^{n} y$ and $D^{n-1} y$ we may show as in the first part that $y_{n-k}$ is eventually negative for any $k=2, \cdots, n$ which contradicts the positivity of $y(t)$.

Using the same technique as above, it follows that if any two consecutive $y_{k}, k \geqq 1$ are ultimately positive, then all the preceding $y_{k}$ are eventually positive. We conclude that a positive solution of (1.1) is necessarily of type $B_{j}$ for some $j=0, \cdots, n-1$. Thus the nonexistence of nonoscillatory solutions of type $B_{j}(0 \leqq j \leqq n-1)$ will imply that all solutions are necessarily oscillatory.

In section two integral criteria are given for the nonexistence of solutions of type $B_{j}$ as well as for the oscillation of all solutions 
of (1.1). Section three provides a necessary and sufficient condition for the existence of a nonscillatory solution of (1.1) with $r(t) \equiv 1$ having prescribed asymptotic form. Recently Yoshizawa [11, 12] has applied the direct method of Lyapunov to study the oscillatory behavior of solutions of certain nonlinear second order ordinary differential equations. In section four his technique is employed to investigate the nonoscillatory behavior of (1.1). For recent related studies see the papers of Burkowski [2], Gollwitzer [3], Staikos and Petsoulas [7], and Wong [9].

2. In this section integral criteria for the nonexistence of solutions of types $B_{j}(j=0, \cdots, n-1)$ are derived. To obtain these results we shall first prove several lemmas.

LEMMA 2.1. Let $y(t)$ be a solution of (1.1) of type $B_{j}$ where either (i) $n$ is even and $j \leqq(n-2) / 2$ or (ii) $n$ is odd and $j \leqq(n-3) / 2$. Then for all sufficiently large $t$,

$$
t y_{k}(t) \leqq 2(2 j+2-k) y_{k-1}(t), \quad k=1, \cdots, 2 j+1
$$

LEMMA 2.2. Let $y(t)$ be a $B_{j}$-solution of (1.1) where $n$, is odd and $j=(n-1) / 2$. Then for all sufficiently large $t$,

and

$$
t y_{n}(t) \leqq 2 M y_{n-1}(t)
$$

$$
t y_{k}(t) \leqq 2\left[M m^{-1}+(n-k)\right] y_{k-1}(t), \quad k=1, \cdots, n-1
$$

Lemma 2.3. Let $y(t)$ be a $B_{j}$-solution of (1.1) where either (i) $n$ is even and $j \geqq n / 2$ or (ii) $n$ is odd and $j \geqq(n+1) / 2$. Then for all sufficiently large $t$,

(a) $t y_{k}(t) \leqq 2(2 j+2-k) y_{k-1}(t), \quad k=n+1, \cdots, 2 j+1$.

(b) $t y_{n}(t) \leqq 2 M(2 j-n+2) y_{n-1}(t)$, and

(c) $t y_{k}(t) \leqq 2\left[M(2 j-n+2) m^{-1}+(n-k)\right] y_{k-1}(t)$, $k=1, \cdots, n-1$.

The proof of each of the three lemmas is elementary using only integration by parts and the definition of a $B_{j}$-solution. For brevity, we will prove only Lemma 2.3.

Proof. Suppose $y(t)$ is a solution of type $B_{j}$. Then there is a $T_{0}>0$ such that $y_{k}(t)>0,(0 \leqq k \leqq 2 j+1)$ and $y_{2 j+2}(t)<0$ for $t \geqq T_{0}$. Hence, $y_{\tau}(t)>0$ for $t-\tau(t) \geqq T_{0}$, i.e., for $t \geqq T_{0}+T=T_{1}$. Thus, $y_{2 j+1}(t)$ is a decreasing function of $t$ for $t \geqq T_{1}$. Consequently,

$$
y_{2 j}(t) \geqq\left[y_{2 j}(s)\right]_{T_{1}}^{t}=\int_{T_{1}}^{t} y_{2 j+1}(s) d s \geqq\left(t-T_{1}\right) y_{2 j+1}(t) \text {. }
$$


Since $\left(t-T_{1}\right) \geqq t / 2$ for $t \geqq 2 T_{1}$, we have $y_{2 j}(t) \geqq t y_{2 j+1}(t) / 2$ which proves (a) for the case $k=2 j+1$.

We proceed inductively and suppose that for some integer $k$, $n+1<k \leqq 2 j+1$,

$$
\left(t-T_{1}\right) y_{k}(t) \leqq(2 j+2-k) y_{k-1}(t) .
$$

An integration of (2.1) yields

$$
\left[\left(s-T_{1}\right) y_{k-1}(s)\right]_{T_{1}}^{t}-\int_{T_{1}}^{t} y_{k-1}(s) d s=\int_{T_{1}}^{t}\left(s-T_{1}\right) y_{k}(s) d s
$$

so that

$$
\begin{aligned}
\left(t-T_{1}\right) y_{k-1}(t) & \leqq[2 j+2-(k-1)] \int_{T_{1}}^{t} y_{k-1}(s) d s \\
& \leqq[2 j+2-(k-1)] y_{k-2}(t),
\end{aligned}
$$

which proves (a). Specifically, for $k=n+1$, (2.1) becomes

$$
\left(t-T_{1}\right) y_{n+1}(t) \leqq(2 j-n+1) y_{n}(t) .
$$

Integrating this by parts results in

$$
\begin{aligned}
\left(t-T_{1}\right) y_{n}(t)-\int_{T_{1}}^{t} y_{n}(s) d s= & \int_{T_{1}}^{t}\left(s-T_{1}\right) y_{n+1}(s) d s \\
& \leqq(2 j-n+1) \int_{T_{1}}^{t} y_{n}(s) d s .
\end{aligned}
$$

Thus, for $t \geqq T_{1}$ we obtain

$$
\begin{aligned}
\left(t-T_{1}\right) y_{n}(t) & \leqq(2 j-n+2) \int_{T_{1}}^{t} y_{n}(s) d s \\
& \leqq(2 j-n+2) M y_{n-1}(t)
\end{aligned}
$$

As in (a) the estimate for $t \geqq 2 T_{1}$ becomes

$$
t y_{n}(t) \leqq 2(2 j-n+2) M y_{n-1}(t)
$$

which proves (b).

Now integration of (2.2) by parts establishes the anchor for an inductive proof of (c).

$$
\begin{aligned}
{\left[m\left(s-T_{1}\right) y_{n-1}(s)\right]_{T_{1}}^{t}-m \int_{T_{1}}^{t} y_{n-1}(s) d s } & =m \int_{T_{1}}^{t}\left(s-T_{1}\right) D^{n} y(s) \\
& \leqq \int_{T_{1}}^{t}\left(s-T_{1}\right) y_{n}(s) d s \\
& \leqq(2 j-n+2) M \int_{T_{1}}^{t} y_{n-1}(s) d s .
\end{aligned}
$$

Thus, for $t \geqq 2 T_{1}$, we have 


$$
\begin{aligned}
m t y_{n-1}(t) / 2 & \leqq m\left(t-T_{1}\right) y_{n-1}(t) \leqq[(2 j-n+2) M+m] \int_{T_{1}}^{t} y_{n-1}(s) d s \\
& \leqq[(2 j-n+2) M+m] y_{n-2}(t),
\end{aligned}
$$

which proves (c) in the case $k=n-1$. Now assume inductively that

$$
\left(t-T_{1}\right) y_{k}(t) \leqq\left[M(2 j-n+2) m^{-1}+(n-k)\right] y_{k-1}(t)
$$

for some $k, 1<k \leqq n-1$. Then

$$
\begin{aligned}
(t- & \left.T_{1}\right) y_{k-1}(t)-\int_{T_{1}}^{t} y_{k-1}(s) d s \\
& =\int_{T_{1}}^{t}\left(s \cdot T_{1}\right) y_{k}(s) d s \\
& \leqq\left[M(2 j-n+2) m^{-1}+(n-k)\right] \int_{T_{1}}^{t} y_{k-1}(s) d s
\end{aligned}
$$

so that for $t \geqq T_{1}$

$$
\left(t-T_{1}\right) y_{k-1}(t) \leqq\left[M(2 j-n+2) m^{-1}+(n-k+1)\right] y_{k-2}(t)
$$

and for $t \leqq 2 T_{1}$

$$
t y_{k-1}(t) \leqq 2\left[M(2 j-n+2) m^{-1}+(n-k+1)\right] y_{k-2}(t)
$$

which proves (c).

We remark that if $y(t)$ is a $B_{j}$-solution of $(1.1)$ on $[0, \infty)$ with $r(t) \equiv 1$ and $\tau(t) \equiv 0$, we may take $T_{1}=0$ and $m=M=1$. The above proof will yield

$$
t y_{2 j+2-k} \leqq k y_{2 j+1-k}
$$

for $k=1, \cdots, 2 j+1$, which is another form of Kiguradze's lemma [4].

Lemma 2.4. Let $y(t)$ be a solution of (1.1) of type $B_{j}$. Then $y_{2 j}(t-\tau(t)) \sim y_{2 j}(t)$.

Proof. Let $y(t)$ be a $B_{j}$-solution of (1.1). For $j \neq(n-1) / 2$ and $t \geqq T_{1}, y_{\tau}(t)$ and $y_{k}(t), k=0, \cdots, 2 j+1$ are all positive while $y_{2 j+2}(t)<0$. Since $\tau(t) \geqq 0$ and $y_{2 j}(t)$ is an increasing function on $\left(T_{1}, \infty\right), y_{2 j}(t-$ $\tau(t))<y_{2 j}(t)$, so that with the help of Lemma 2.1 or 2.3 , we have

$$
\left|\frac{y_{2 j}(t-\tau(t))}{y_{2 j}(t)}-1\right|=\tau(t) \frac{y_{2 j+1}(s)}{y_{2 j}(t)}<\frac{2 T}{s} \frac{y_{2 j}(s)}{y_{2 j}(t)}<\frac{2 T}{s},
$$

where $t-\tau(t) \leqq s \leqq t$. Since $s$ tends to infinity with $t$, the lemma follows in this case. The case $j=(n-1) / 2$ follows in a similar manner using Lemma 2.2 and the estimate $m D y_{2 j}(s) \leqq y_{2 j+1}(s)$. 
We remark that this lemma is analogous to one proved by Bradley [1] for the linear equation

$$
y^{\prime \prime}(t)+p(t) y_{\tau}(t)=0 \text {. }
$$

His proof can be modified to yield Lemma 2.4. We note that Lemmas $2.1,2.2$, and 2.3 are valid for unbounded $t$, provided $\lim _{t \rightarrow \infty}(t-\tau(t))=$ $+\infty$. A weaker version of Lemma 2.4 is also true for unbounded $\tau(t)$, provided $0 \leqq \tau(t) \leqq \ell t$, where $\mu$ can be specified. In general, if $y(t)$ is a $B_{j}$-solution of (1.1) described by Lemmas 2.1 or 2.3 , then we may take $\mu<1 / 2$; otherwise the stronger estimate $\mu<m /(M+m)$ is required. The conclusion of Lemma 2.4 is changed to read: There are constants $k_{j}>0$ and $t_{j}>0$ such that $y_{2 j}(t-\tau(t))>k_{j} y_{2 j}(t)$ for $t>t_{j}$. With these lemmas we can now give criteria for the nonexistence of solutions of type $B_{j}(0 \leqq j \leqq n-1)$.

TheOREM 2.5. Suppose that for all constants $C>0$ and some $j=0, \cdots,(n-1)$,

$$
\int^{\infty} t^{2 j} f\left(t, C t^{2 j}\right) d t=+\infty .
$$

Then (1.1) has no solutions of type $B_{j}$.

Proof. Let $y(t)$ be a positive solution of type $B_{j}$ and let $w(t)=$ $y_{2 n-1}(t) / y_{2 j}(t)$. Then (1.1) shows that

$$
w^{\prime}(t)+y_{2 n-1}(t) D y_{2 j}(t) y_{2 j}^{-2}(t)+y_{\tau}(t) f\left(t, y_{\tau}(t)\right) y_{2 j}^{-1}(t)=0 \text {. }
$$

For $j \neq(n-1) / 2, D y_{2 j}(t)=y_{2 j+1}(t)>0$; if $n$ is odd and $j=(n-1) / 2$, $D y_{2 j}(t)=y_{2 j+1}(t) / r(t)>0$ since $r(t)>0$. Since $y_{2 n-1}(t)$ and $y_{2 j}(t)$ are positive for $t>T_{1}$, this reduces to

$$
w^{\prime}(t)+y_{\tau}(t) f\left(t, y_{\tau}(t)\right) y_{2 j}^{-1}(t)<0, t \geqq T_{1} .
$$

There are three cases to consider

(i) $n$ is even and $j \leqq(n-2) / 2$ or $n$ is odd and $j \leqq(n-3) / 2$;

(ii) $n$ is odd and $j=(n-1) / 2$; and

(iii) $n$ is even and $j \geqq n / 2$ or $n$ is odd and $j \geqq(n+1) / 2$. Applying Lemmas 2.1, 2.2, and 2.3 to cases (i), (ii), and (iii) respectively, we obtain

$$
t^{2 j} y_{2 j}(t) \leqq M_{j} y(t)
$$

where

$$
\begin{gathered}
2^{2 j}(2 j+1) ! \\
M_{j}=2^{2 j} \prod_{k=1}^{2 j}\left[M m^{-1}+(n-k)\right],
\end{gathered}
$$

case (i), case (ii), 


$$
2^{2 j}(2 j-n+2) ! M \prod_{k=1}^{n-1}\left[(2 j-n+2) M m^{-1}+(n-k)\right], \quad \text { case (iii). }
$$

Letting $N_{j}=M_{j}^{-1}$, we have $y(t) \geqq N_{j} t^{2 j} y_{2 j}(t)$ for $t \geqq 2 T_{1}$, so for $t \geqq 2 T_{1}+T=T_{2}$ we have

$$
y_{\tau}(t) \geqq N_{j}(t-\tau(t))^{2 j} y_{2 j}(t-\tau(t)) \geqq N_{j}(t-T)^{2 j} y_{2 j}(t-\tau(t))
$$

because $0 \leqq \tau(t) \leqq T$. Since $D y_{2 j}(t)>0$ for $t \geqq T_{1}$, there is a constant $C_{0}$ such that $y_{2 j}(t) \geqq C_{0}$ for $t \geqq T_{1}$. Hence, $y_{2 j}(t-\tau(t)) \geqq C_{0}$ for $t \geqq T_{1}+T$. Moreover, $(t-T) \geqq t / 2$ for $t \geqq 2 T$. Because $T_{2}=2 T_{1}+$ $T>\max \left(T_{1}+T, 2 T\right)$, both estimates hold for $t \geqq T_{2}$. Combining the above estimates with (2.4) via hypothesis (ii) and using Lemma 2.4, we arrive at

$$
w^{\prime}(t)+N K t^{2 j} f\left(t, C t^{2 j}\right) \leqq 0, t \geqq T_{3}
$$

for some $T_{3} \geqq T_{2}$, where $N=N_{j} 2^{-2 j}, C=2^{-2 j} N_{j} C_{0}$ and $K=K_{j}>0$ is the constant from Lemma 2.4. An integration of this together with (2.3) shows that $w(t)$ is eventually negative, which is absurd.

Since the divergence of $t^{2 j} f\left(t, C t^{2 j}\right)$ implies that of $t^{2(j+1)} f\left(t, C t^{2(j+1)}\right)$ the conclusion of Theorem 2.5 may be strengthened to exclude solutions of type $B_{k}$ where $j \leqq k \leqq n-1$. The theorem may also be restated as follows.

THEOREM 2.6. Suppose (2.3) holds for all constants $C>0$ and for some $j=0, \cdots, n-1$. Then either (1.1) is oscillatory or else $y(t) y_{2 j}(t)<0$ for $t$ sufficiently large.

For $j=n-1$ and $r(t) \equiv 1$, Theorem 2.6 reduces to the alternative that either (1.1) is oscillatory or else $y(t) D^{2 n-2} y(t)<0$ for $t$ sufficiently large, which is essentially Theorem 3.1 of Ladas [5].

Moreover, in view of the fact that all positive solutions are of types $B_{j}$ for some $j(0 \leqq j \leqq n-1)$, we can immediately restate Theorem 2.5 as a criterion of oscillation.

COROLLARY 2.7. If for all constants $C>0$

$$
\int^{\infty} f(t, C) d t=+\infty,
$$

then all solutions of (1.1) are oscillatory.

COROLLARY 2.8. Suppose $p(t)$ is continuous and eventually positive and that

$$
\int^{\infty} p(t) d t=+\infty
$$


Then all solutions of the equation

$$
D^{n}\left[r(t) D^{n} y(t)\right]+p(t) y_{\tau}^{2 \lambda+1}(t)=0, \lambda>0
$$

are oscillatory.

The conclusion of Corollary 2.8 is true in the case $\lambda=0$ in (2.5). In this instance, (2.5) is not a special case of (1.1) since $f(t, u)=p(t)$ does not satisfy hypothesis (ii) of section one. To permit this extension, we may suppose that $y(t)$ is a $B_{j}$-solution of (2.5) and let $w(t)=y_{2 n-1}(t) / y_{2 j}(t)$. Equation (2.4) becomes

$$
w^{\prime}(t)+p(t) y_{\tau}(t) y_{2 j}^{-1}(t)<0, t \geqq T_{1} .
$$

Applying Lemmas 2.1-2.4, which are independent of hypothesis (ii), produces the same contradiction as in the proof of Theorem 2.5. Thus, if $\lambda=0$ and

$$
\int^{\infty} t^{2 j} p(t) d t=\infty
$$

for some $j=0, \cdots, n-1$, (2.5) has no $B_{k}$-solutions for $k=j, \cdots$, $n-1$. Corollary 2.8 then follows by specifying $j=0$.

Note. When $n=2$ and $j=1$, Lemma 2.3 reduces to Lemma 2.1 of Terry and Wong [8]. Similarly, letting $n=2$ and $j=0,1$ in Lemma 2.4, we obtain Lemma 2.2 (a), (b) of [8]. Moreover, Theorem 2.5, Corollary 2.7, and Corollary 2.8 here are, respectively, the analogues of Theorem 2.8, Theorem 2.4, and Corollary 2.5 of [8].

3. In this section an asymptotic result is established for the equation

$$
D^{2 n} y(t)+f\left(t, y_{\tau}(t)\right) y_{\tau}(t)=0,
$$

where $f(t, u)$ satisfies the three conditions of section one.

LEMMA 3.1. Let $y(t)$ be a solution of (3.1) which is eventually positive. Then

$$
D^{2 n-1} y(t) \sim(2 n-1) ! t^{1-2 n} y(t)
$$

Proof. Suppose $y(t)$ is a solution of (3.1) such that $y(t)>0$ for $t \geqq T_{1}$. Then $y_{\tau}(t)>0$ for $t-\tau(t) \geqq T_{1}$, i.e., for $t \geqq T_{1}+T=T^{*}$. By Taylor' theorem with remainder, for $t \geqq T^{*}$

$$
(2 n-1) ! R(t)=(2 n-1) ! y(t)+\int_{T^{*}}^{t}(t-s)^{2 n-1} y_{\tau}(s) f\left(s, y_{\tau}(s)\right) d s,
$$

where 


$$
R(t)=\sum_{k=0}^{2 n-1} \frac{1}{k !} D^{k} y\left(T^{*}\right)\left(t-T^{*}\right)^{k} .
$$

Since $y_{\tau}(s)$, and hence $-D^{2 n} y(s)$, is positive for $s>T^{*}$, condition (iii) together with $\left(t-T^{*}\right)>(t-s)>0$ imply that

$$
\begin{aligned}
& (2 n-1) ! R(t) \\
& \quad \leqq(2 n-1) ! y(t)+\left(t-T^{*}\right)^{2 n-1}\left[D^{2 n-1} y\left(T^{*}\right)-D^{2 n-1} y(t)\right] .
\end{aligned}
$$

Dividing this by $\left(t-T^{*}\right)^{2 n-1}$ and noting that

$$
\lim _{t \rightarrow \infty}(2 \mathrm{n}-1) !\left(t-T^{*}\right)^{1-2 n} R(t)=D^{2 n-1} y\left(T^{*}\right),
$$

it follows upon passage to the limit that

$$
\begin{aligned}
& D^{2 n-1} y\left(T^{*}\right) \\
& \quad \leqq \varliminf_{t \rightarrow \infty}(2 n-1) !\left(t-T^{*}\right)^{1-2 n} y(t)+D^{2 n-1} y\left(T^{*}\right)-\lim _{t \rightarrow \infty} D^{2 n-1} y(t) .
\end{aligned}
$$

Hence,

$$
\lim _{t \rightarrow \infty} D^{2 n-1} y(t) \leqq \varliminf_{t \rightarrow \infty}(2 n-1) !\left(t-T^{*}\right)^{1-2 n} y(t) .
$$

To prove the reverse inequality let $\sigma$ be chosen such that $T^{*}<\sigma<t$. By restricting $s$ to lie in the interval $\left[T^{*}, \sigma\right]$, we have $(t-s)^{2 n-1} \geqq(t-\sigma)^{2 n-1}$ and

$$
\begin{aligned}
(2 n-1) ! R(t) & \geqq(2 n-1) ! y(t)+(t-\sigma)^{2 n-1} \int_{T^{*}}^{\sigma} y_{\tau}(s) f\left(s, y_{\tau}(s)\right) d s \\
& =(2 n-1) ! y(t)+(t-\sigma)^{2 n-1}\left[D^{2 n-1} y\left(T^{*}\right)-D^{2 n-1} y(\sigma)\right] .
\end{aligned}
$$

Multiplying this by $(t-\sigma)^{1-2 n}$, keeping $\sigma$ fixed and letting $t \rightarrow \infty$ through a sequence of points for which $(t-\sigma)^{1-2 n} y(t)$ tends to its limit superior, we obtain

$$
\begin{aligned}
& D^{2 n-1} y\left(T^{*}\right) \\
& \quad \geqq \varlimsup_{t \rightarrow \infty}(2 n-1) !\left(t-T^{*}\right)^{1-2 n} y(t)+D^{2 n-1} y\left(T^{*}\right)-D^{2 n-1} y(\sigma)
\end{aligned}
$$

from which it follows that

$$
\varlimsup_{t \rightarrow \infty}(2 n-1) !\left(t-T^{*}\right)^{1-2 n} y(t) \leqq D^{2 n-1} y(\sigma) .
$$

Since $\sigma$ is arbitrary and $\lim _{t \rightarrow \infty} D^{2 n-1} y(t)$ exists, it follows that

$$
\varlimsup_{t \rightarrow \infty}(2 n-1) !\left(t-T^{*}\right)^{1-2 n} y(t) \leqq \lim _{t \rightarrow \infty} D^{2 n-1} y(t) .
$$

Combining this with (3.3) yields the desired result.

THEOREM 3.2. Equation (3.1) has a positive solution y(t) satisfying 


$$
\lim _{t \rightarrow \infty} t^{1-2 n} y(t)=k, 0<k<\infty,
$$

if, and only if, for some $C>0$

$$
\int^{\infty} t^{2 n-1} f\left(t, C t^{2 n-1}\right) d t<\infty \text {. }
$$

Proof. Suppose (3.5) holds. A lower limit $T_{0}$ can be chosen sufficiently large so that

$$
\int_{T_{0}}^{\infty} t^{2 n-1} f\left(t, C t^{2 n-1}\right) d t<(2 n-1) !-1 / 2 .
$$

If $y(t)=y\left(t, T_{0}\right)$ is the solution of (3.1) defined by $D^{k} y\left(T_{0}\right)=0, k=0$, $\cdots, 2 n-2, D^{2 n-1} y\left(T_{0}\right)=(2 n-1) ! C$ and $y(t)=0$ for $T_{0}-T \leqq t \leqq T_{1}$, then $y\left(t, T_{0}\right)$ is positive on some open interval whose left endpoint is $T_{0}$. Let $t=T_{1}$ be the first zero of $y\left(t, T_{0}\right)$ in $\left(T_{0}, \infty\right)$. By Taylor's theorem we have

$$
\begin{aligned}
(2 n-1) ! C\left(t-T_{0}\right)^{2 n-1}= & (2 n-1) ! y\left(t, T_{0}\right) \\
& +\int_{T_{1}}^{t}(t-s)^{2 n-1} y_{\tau}(s) f\left(s, y_{\tau}(s)\right) d s .
\end{aligned}
$$

Since $y(s)>0$ for $T_{0}-T \leqq s \leqq T_{1}, y_{\tau}(s)>0$ for $T_{0}-T \leqq s-\tau(s) \leqq T_{1}$, i.e., for $s \geqq T_{0}-T+\tau(s)$. Hence $y_{\tau}(s)>0$ for $s \geqq T_{0}$. A similar argument shows that $y_{\tau}(s)>0$ for $s \leqq T_{1}$. Thus

$$
y(t)=y\left(t, T_{0}\right) \leqq C\left(t-T_{0}\right)^{2 n-1}, T_{0} \leqq t \leqq T_{1} .
$$

Moreover, letting $t=T_{1}$ in (3.6) we have

$$
\begin{aligned}
(2 n-1) ! C\left(T_{1}-T_{0}\right)^{2 n-1} & =\int_{T_{0}}^{T_{1}}\left(T_{1}-s\right)^{2 n-1} y_{\tau}(s) f\left(s, y_{\tau}(s)\right) d s \\
& \leqq\left(T_{1}-T_{0}\right)^{2 n-1} \int_{T_{0}}^{T_{1}} y_{\tau}(s) f\left(s, y_{\tau}(s)\right) d s .
\end{aligned}
$$

By condition (iii) and (3.7)

$$
y_{\tau}(s) f\left(s, y_{\tau}(s)\right) \leqq C s^{2 n-1} f\left(s, C s^{2 n-1}\right),
$$

so that (3.8) yields

$$
(2 n-1) ! \leqq \int_{T_{0}}^{T_{1}} s^{2 n-1} f\left(s, C s^{2 n-1}\right) d s,
$$

which contradicts $\left(3.5^{\prime}\right)$ and demonstrates the existence of a positive solution satisfying (3.4).

To prove necessity let $y(t)$ be a positive solution of (3.1) satisfying (3.4). It follows from Lemma 3.1 that 


$$
\int_{T_{1}}^{\infty} y_{\tau}(s) f\left(s, y_{\tau}(s)\right) d s=D^{2 n-1} y\left(T_{1}\right)-(2 n-1) ! k
$$

(3.4) shows that for any $\varepsilon>0$ there is a $T_{*} \geqq T_{1}$ such that $y(t) \geqq(k-\varepsilon) t^{2 n-1}$ for all $t \geqq T_{*}$ so that $(k-\varepsilon)(t-T)^{2 n-1} \leqq y_{\tau}(t)$ for $t>T^{*}=T_{*}+T$. Also, it follows from (ii) that

$$
f\left(s, y_{\tau}(s)\right) \geqq f\left[s,(k-\varepsilon)(s-T)^{2 n-1}\right] .
$$

Since (3.9) is valid with $T_{1}$ replaced by $T^{*}$,

$$
\begin{aligned}
& D^{2 n-1} y\left(T^{*}\right)-(2 n-1) ! k \\
& \quad \geqq(k-\varepsilon) \int_{T^{*}}^{\infty}(s-T)^{2 n-1} f\left[s,(k-\varepsilon)(s-T)^{2 n-1}\right] d s .
\end{aligned}
$$

For so $s \geqq 2 T$,

$$
\begin{aligned}
& \int^{\infty} s^{2 n-1} f\left(s, C s^{2 n-1}\right) d s<2^{2 n-1} \\
& \quad \times \int^{\infty}(s-T)^{2 n-1} f\left[s, 2^{2 n-1} C(s-T)^{2 n-1}\right] d s \leqq N_{1},
\end{aligned}
$$

where $N_{1}=2^{2 n-1}(k-\varepsilon)^{-1}\left[D^{2 n-1} y\left(T^{*}\right)-(2 n-1) ! k\right]$ and $C=(k-\varepsilon) / 2^{2 n-1}$. The conclusion follows.

For the linear equation (2.5) with $\lambda=0, n=2, r(t) \equiv 1$, and $\tau(t) \equiv 0$, this result reduces to that of Leighton and Nehari [6]. As in the discussion following Corollary 2.8, we observe that when $\lambda=0$ and $r(t) \equiv 1,(2.5)$ is not a special case of (3.1). However, Theorem 3.2 remains valid and the proof given above may be easily modified to yield the result. The details of this are omitted for brevity. For the nonlinear equation (3.1) with $\tau(t) \equiv 0$ and $n=2$, see also Wong [10]. The case of (3.1) with $\tau(t) \not \equiv 0$ and $n=2$ is treated by Terry and Wong [8].

4. In this section we shall apply the direct method of Lyapunov to obtain nonoscillation criteria for (1.1). For convenience, we first introduce some notation which will be used in the section. Let $R_{a}=[a, \infty), a \geqq 0, R^{*}=(0, \infty), R_{*}=(-\infty, 0)$ and $R^{1}=R=(-\infty, \infty)$. We shall abbreviate the cartesian products of these intervals as follows:

$$
\begin{aligned}
R^{p^{*}} & =R^{*} \times R^{*} \times \cdots \times R^{*}, p \text { times } \\
R_{p^{*}} & =R_{*} \times R_{*} \times \cdots \times R_{*}, p \text { times } \\
R_{*}{ }^{*} & =R_{*} \times R^{*} ; R^{*}{ }_{*}=R^{*} \times R_{*} .
\end{aligned}
$$

Other products may be defined in terms of these, e.g.,

$$
R_{a}^{p^{*}}=R_{a} \times R^{p^{*}} .
$$


To begin with we shall consider an arbitrary $2 n$th order equation of the form

$$
D^{n}\left[r(t) D^{n} y(t)\right]+F\left(t, \sigma(t), \sigma_{\tau}(t)\right)=0,
$$

where

$$
\sigma(t)=\left(y_{0}(t), \cdots, y_{2 n-1}(t)\right) \text { and } \sigma_{\tau}(t)=\sigma(t-\tau(t)) .
$$

A real-valued function $V(t, \sigma)$ will be called a Lyapunov function if $V(t, \sigma)$ is continuous in its domain and locally Lipschitzian in $\sigma$. Following Yoshizawa $[11,12]$, we define the trajectory derivative $\dot{V}_{(1)}$ of $V$ along solutions $\sigma(t)$ of (4.1) by

$$
\dot{V}_{(1)}(t, \sigma)=\varlimsup_{h \downarrow 0} \frac{1}{h}[V(t+h, \sigma(t+h))-V(t, \sigma(t))] .
$$

The first result of this section is an extension of Yoshizawa's theorem to (4.1) and the proof is based on his.

THEOREM 4.1. Let $V$ be a real-valued continuous function defined on $R_{a}^{(2 j+2) *} \times\left(R_{*}^{*}\right)^{n-j-1}$ for some $a>0$ such that

(i) $V$ tends to infinity uniformly for $\sigma \in R^{(2 j+2) *} \times\left(R_{*}^{*}\right)^{n-j-1}$ as $t$ tends to infinity; and

(ii) for each solution $y(t)$ of (4.1) such that $(t, \sigma(t)) \in R_{b}^{(2 j+2) *} \times$ $\left(R_{*}^{*}\right)^{n-j-1}$ for some $b \geqq a, \dot{V}_{(1)}(t, \sigma(t)) \leqq 0$.

Then (4.1) has no solution of type $B_{j}$.

Proof. Let $y(t)$ be a solution of type $B_{j}$. There is a positive $a$ for which $\sigma(t) \in R_{a}^{(2 j+2) *} \times\left(R_{*}^{*}\right)^{n-j-1}$ if $t \geqq a$. By (ii) for $t$ sufficiently large, i.e., for $t \geqq b \geqq a$,

$$
V(t, \sigma(t)) \leqq V(b, \sigma(b)) .
$$

On the other hand, (i) implies that there is a $c \geqq b$ for which

$$
V(t, \sigma(t))>V(b, \sigma(b))
$$

if $t>c$, which is a contradiction.

As in other applications of the direct method of Lyapunov, the key to applying this result is the construction of suitable Lyapunov functions $V$ having the requisite properties. In this case of (1.1) Theorem 2.5 may be regarded as a special case of this result, for if $y(t)$ is a $B_{j}$-solution there are constants $C>0$ and $K>0$ such that for sufficiently large $t, y_{\tau}(t) \geqq N K t^{2 j} y_{2 j}(t) \geqq C t^{2 j}$. It follows that by taking a suitable $T^{*}$ the function

$$
V(t, \sigma(t))=y_{2 n-1}(t)\left[y_{2 j}(t)\right]^{-1}+N K \int_{T^{*}}^{t} s^{2 j} f\left(s, C s^{2 j}\right) d s
$$


will be a Lyapunov function satisfying the conditions of Theorem 4.1 provided (2.4) holds.

Another application of Theorem 4.1 to equation (4.1) is given by the following result.

THEOREM 4.2. Suppose there exist functions $p(t)$ and $\rho(u)$ of class $C\left(R_{a}\right)$ and $C^{1}(R)$ respectively such that

(i) $\rho^{\prime}(u) \geqq 0 ; u \rho(u)>0, u \neq 0$;

(ii) $F(t, \sigma(t)) \geqq p(t) \rho(y), y \geqq 0$;

(iii) $\int^{\infty} p(t) d t=+\infty$.

Then (4.1) has no solutions of type $B_{j}$.

Proof. Suppose $y(t)$ is a solution of (4.1) of type $B_{j}$. Then $(t, \sigma(t)) \in R_{b}^{(2 j+2) *} \times\left(R_{*}{ }^{*}\right)^{n-j-1}$ for some $b \geqq a$. Let $V(t, \sigma)$ be the function defined by

$$
V(t, \sigma(t))=y_{2 n-1}(t)[\rho(y(t))]^{-1}+\int_{b}^{t} p(s) d s .
$$

In view of hypotheses (i), (ii), and (iii), $V$ will clearly satisfy condition (i) of Theorem 4.1. Moreover, since both $y^{\prime}(t)$ and $y_{2 n-1}(t)$ are positive for large $t$, a simple calculation with the help of (i) and (ii) shows that

$$
\begin{aligned}
\dot{V}_{(1)} & =\frac{D y_{2 n-1}(t)}{\rho[y(t)]}+p(t)-\frac{\rho^{\prime}[y(t)]}{\rho^{2}[y(t)]} y_{2 n-1}(t) y^{\prime}(t) \\
& \leqq p(t)-\frac{F(t, \sigma(t))}{\rho[y(t)]} \leqq 0 .
\end{aligned}
$$

Theorem 4.1 is thus applicable and we conclude that (4.1) cannot have any solution of type $B_{j}$.

Acknowledgment. This paper is part of a thesis submitted to the Graduate School of Michigan State University in partial fulfillment of the requirements for the degree of Doctor of Philosophy. The author wishes to thank Dr. Pui-Kei Wong for his guidance during the writing of the thesis.

\section{REFERENCES}

1. J. S. Bradley, Oscillation theorems for a second order delay equation, J. Differential Equations, 8 (1970), 397-403.

2. F. Burkowski, Oscillation theorems for a second order nonlinear functional differential equation, J. Math. Anal. Appl., 33 (1971), 258-262.

3. H. E. Gollwitzer, On nonlinear oscillations for a second order delay equation, J. Math. Anal. Appl., 26 (1969), 385-389. 
4. I. T. Kiguradze, Oscillation properties of solutıons of certain ordinary differential equations, Dokl. Akad. Nauk SSSR, 144 (1962), 33-36; translated in Soviet Math. Dokl., 3 (1962), 649-652.

5. G. Ladas, Oscillation and asymptotic behavior of solutions of differential equations with retarded arguments, J. Differential Equations, 10 (1971), 281-290.

6. W. Leighton and Z. Nehari, On the oscillation of solutions of self-adjoint linear differential equations of the fourth order, Trans. Amer. Math. Soc., 89 (1958), 325377.

7. V. A. Staikos and A. G. Petsoulas, Some oscillation criteria for second order nonlinear delay differential equations, J. Math. Anal. Appl., 30 (1970), 695-701.

8. R. D. Terry and P. K. Wong, Oscillatory properties of a fourth order delay differential equation, Funkcialaj Ekvacioj, 15 (1972), 209-221.

9. J. S. W. Wong, Second order oscillation with retarded argumenes, Proceedings of Conference on Ordinary Differential Equations, June 14-23, (1971), edited by L. Weiss, Academic Press, New York, 1972, 581-596.

10. P. K. Wong, On a class of nonlinear fourth order differential equations, Ann. Mat. Pura Appl., 81 (1969), 331-346.

11. T. Yoshizawa, Oscillatory property of solutions of second order differential equations, Tohoku Math. J., 22 (170), 619-634.

12. - Oscillatory property for second order differential equations, Proceedings of Conference on Ordinary Differential Equations, June 14-23, (1971), edited by L. Weiss, Academic Press, New York, 1972, 315-327.

Received December 18, 1972 and in revised form July 23, 1973.

Georgia Institute of Technology 


\section{PACIFIC JOURNAL OF MATHEMATICS}

EDITORS

RICHARD ARENS (Managing Editor)

University of California

Los Angeles, California 90024

R. A. Beaumont

University of Washington

Seattle, Washington 98105

J. DugundJI

Department of Mathematics

University of Southern California

Los Angeles, California 90007

D. Gilbarg and J. Milgram

Stanford University

Stanford, California 94305

\section{ASSOCIATE EDITORS}

E. F. BECKENBACH

B. H. NeumanN

F. WOLF

K. YOSHIDA

\section{SUPPORTING INSTITUTIONS}

UNIVERSITY OF BRITISH COLUMBIA CALIFORNIA INSTITUTE OF TECHNOLOGY

UNIVERSITY OF CALIFORNIA

MONTANA STATE UNIVERSITY

UNIVERSITY OF NEVADA

NEW MEXICO STATE UNIVERSITY

OREGON STATE UNIVERSITY

UNIVERSITY OF OREGON

OSAKA UNIVERSITY

\author{
UNIVERSITY OF SOUTHERN CALIFORNIA \\ STANFORD UNIVERSITY \\ UNIVERSITY OF TOKYO \\ UNIVERSITY OF UTAH \\ WASHINGTON STATE UNIVERSITY \\ UNIVERSITY OF WASHINGTON \\ $\stackrel{*}{*} \stackrel{*}{*}{ }^{*}{ }^{*}{ }^{2}$ AMERICAN MATHEMATICAL SOCIETY \\ NAVAL WEAPONS CENTER
}




\section{Pacific Journal of Mathematics}

\section{Vol. 52, No. $1 \quad$ January, 1974}

David R. Adams, On the exceptional sets for spaces of potentials ............ 1

Philip Bacon, Axioms for the Čech cohomology of paracompacta ............ 7

Selwyn Ross Caradus, Perturbation theory for generalized Fredholm operators ..... 11

Kuang-Ho Chen, Phragmén-Lindelöf type theorems for a system of nonhomogeneous equations ............................ 17

Frederick Knowles Dashiell, Jr., Isomorphism problems for the Baire classes .......

M. G. Deshpande and V. K. Deshpande, Rings whose proper homomorphic images are right subdirectly irreducible . . . . . . . . . . . . . . . . . . . . . . . . .

Mary Rodriguez Embry, Self adjoint strictly cyclic operator algebras .............

Paul Erdős, On the distribution of numbers of the form $\sigma(n) / n$ and on some related

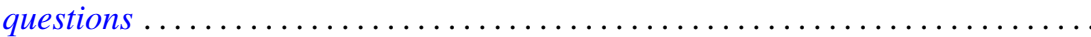

Richard Joseph Fleming and James E. Jamison, Hermitian and adjoint abelian

operators on certain Banach spaces ............................

Stanley P. Gudder and L. Haskins, The center of a poset .................. 85

Richard Howard Herman, Automorphism groups of operator algebras . . . ........

Worthen N. Hunsacker and Somashekhar Amrith Naimpally, Local compactness of families of continuous point-compact relations ....................

Donald Gordon James, On the normal subgroups of integral orthogonal groups ....

Eugene Carlyle Johnsen and Thomas Frederick Storer, Combinatorial structures in

loops. II. Commutative inverse property cyclic neofields of prime-power

order.

Ka-Sing Lau, Extreme operators on Choquet simplexes . . . . . . . . . . . . . . 129

Philip A. Leonard and Kenneth S. Williams, The septic character of 2, 3, 5 and $7 \ldots 143$

Dennis McGavran and Jingyal Pak, On the Nielsen number of a fiber map ........ 149

Stuart Edward Mills, Normed Köthe spaces as intermediate spaces of $L_{1}$ and

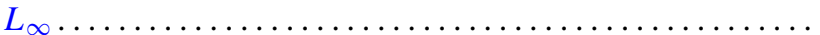

Philip Olin, Free products and elementary equivalence. .

Louis Jackson Ratliff, Jr., Locally quasi-unmixed Noetherian rings and ideals of the principal class.

Seiya Sasao, Homotopy types of spherical fibre spaces over spheres ...

Helga Schirmer, Fixed point sets of polyhedra ...

Kevin James Sharpe, Compatible topologies and continuous irreducible

representations.

Frank Siwiec, On defining a space by a weak base . . . . . . . . . . . . . . . 233

James McLean Sloss, Global reflection for a class of simple closed curves ....... 247

M. V. Subba Rao, On two congruences for primality . .

Raymond D. Terry, Oscillatory properties of a delay differential equation of even

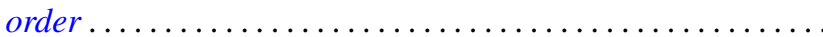

Joseph Dinneen Ward, Chebyshev centers in spaces of continuous functions . .

Robert Breckenridge Warfield, Jr., The uniqueness of elongations of Abelian

groups...

V. M. Warfield, Existence and adjoint theorems for linear stochastic differential

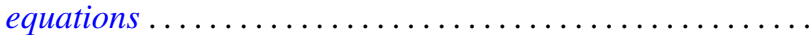

